\title{
Efficacy of IMOD in the treatment of oral lichen planus
}

\author{
-Efficacy of IMOD in oral lichen planus
}

Farzaneh Agha-Hosseini ${ }^{1}$, Iraj Mirzaii-Dizgah ${ }^{2}$, Mohammad Abdollahi $^{3}$, Neda Akbari-Gillani ${ }^{1}$

\author{
${ }^{1}$ Department of Oral Medicine/Dental Research Center, Dentistry School, Tehran University of Medical Sciences, Tehran, Iran; \\ ${ }^{2}$ Department of Physiology, School of Medicine, AJA University of Medical Sciences, Tehran, Iran; \\ ${ }^{3}$ Pharmaceutical Sciences Research Center, Tehran University of Medical Sciences, Tehran, Iran. \\ E-mail: aghahose@sina.tums.ac.ir; emirzaii@razi.tums.ac.ir; $\underline{\operatorname{mohammad} @ \text { tums.ac.ir }}$
}

Received 17 March 2011; revised 26 April 2011, accepted 13 May 2011.

\begin{abstract}
Objectives: Oral lichen planus (OLP) is a chronic, immunologically mediated, mucocutaneous disorder. A wide range of topical and systemic therapies have been used in the treatment of OLP. The efficacy of IMOD (an Iranian new immunomodulator drug, containing selenium, carotene, and flavonoids) in the management of oral lichen planus was evaluated. Study design: In a before-after clinical trial study, thirty patients (21 women and 9 men; age range 35 66 years with 112 lesions) with lichen planus were enrolled. The study covered a three-month period of therapy by IMOD (400 mg/day) and a three-month follow-up period after drug cessation. Outcome measures include soreness relief based on the "nu-meric scale", and clinical improvement of lesion size and score. Saliva levels of TNF- $\alpha$ was analysed at the baseline and after treatment by ELISA. Statistical analysis of Wilcoxon and paired student's t-test were used. Results: Approximately $85 \%$ of patients showed partial to complete improvement and re-mained symptom free after drug cessation. There was no significant difference in mean saliva TNF- $\alpha$ level before and after the treatment. Conclusion: These results suggest that IMOD seems to be an effective alternative treatment for OLP and TNF- $\alpha$ may not be a good indicator for monitoring therapeutic response of OLP.
\end{abstract}

Keywords: Oral Lichen Planus; IMOD; TNF- $\alpha$

\section{INTRODUCTION}

Oral lichen planus (OLP) is a chronic, immunologically mediated, mucocutaneous disorder [1]. Prevalence rate reported for OLP varies between $0.5 \%$ and $2.2 \%$ in the literature, and the mean age at the time of diagnosis is approximately 55 years. Despite the self-limiting nature of cutaneous lesions, oral lesions are chronic and rarely undergo spontaneous remission, and are potentially premalignant. World Health Organization has categorized OLP as a pre-cancerous condition, which is "a general state associated with a significant increased risk of cancer". The pathogenesis of OLP is unclear. It was shown that OLP patients have more cellular lipid peroxidation [2]. Both antigen specific and non-specific mechanisms maybe involved. Antigen specific pathways include a cell mediated immunological response to an induced antigenic change in the oral mucosa [1], in which cytotoxic CD8+ $\mathrm{T}$ cells trigger the apoptosis of epithelial cells following formation of autoreactive $\mathrm{T}$ lymphocytes [3].

A wide range of topical and systemic therapies have been used in the treatment of OLP. Since the etiology behind OLP is unknown, basic conditions are lacking for development of preventive therapies. Several topical drugs have been suggested, including steroids, calcineurin inhibitors (cyclosporine and tacrolimus), retinoid and ultraviolet phototherapy [3]. Among these, topical steroids are widely used and have been accepted as the first choice treatment [3], because they have anti- inflammatory and anti-immunologic effects at the same time. But their prolonged use should be avoided, because of the associated adverse effect such as candidiasis over infection, hypothalamic-pituitary-adrenal axis suppression, skin fragility and telangectasis, and lesion recurrence after treatment cessation [4].

IMOD is an Iranian new immunomodulator drug (international publication number: WO 2007/087825A1) with herbal origin. It is produced from a combination of ethanolic Rosa sp Urtica Dicica and Tanacetum Vulgare extracts, contains selenium, carotene, flavonoids and urea, and having been exposed to a pulsed electromagnetic field. The immunomodulatory mechanisms of IMOD are not clearly understood, but it can increase CD4 + lymphocyte count after a course of treatment of 1 to 3 months. Its effect is also preserved for a long period after cessa- 
tion of treatment. It has passed 3 phases of clinical trial and there were no adverse effects found. Pregnancy and breast feeding is the only contra-indications of this drug. Considering the durability, safety and immunologic effects, it could be used in treatment of immunologic disorders [5].

Serum or saliva level of TNF- $\alpha$ has been used in some studies for monitoring the therapeutic response of OLP $[6,7]$. TNF- $\alpha$ is a multi-functional cytokine that mediates inflammation, immune response, and apoptosis, and also has a significant role in normal development and homeostasis of several organs [8]. Positive statistical correlation exists between TNF- $\alpha$ level in serum and saliva. On the other hand, saliva collection is non-aggressive and more cost effective.

The purpose of this study was to evaluate the efficacy of IMOD in the relief of symptoms and signs of OLP lesion. We have also measured the level of TNF- $\alpha$ in unstimulated whole saliva before and after treatment.

\section{MATERIAL AND METHOD}

This was an open label before-after clinical trial study enrolling 30 patients ( 21 females and nine males). The study cases were selected from patients referred to the Oral Medicine Department, Faculty of Dentistry, Tehran University of Medical Sciences.

Inclusion criteria were the presence of symmetric reticular or papular lesions with or without erosive/atrophic components proven by biopsy. Histopathological diagnosis of OLP (Presence of well-defined band like zones of inflammatory infiltration confined to the superficial part of the connective tissue, consisting mainly of mature lymphocytes - vacuolar alteration of the basal layer of the epithelium), was based on "a modified definition of the World Health Organization" [9]. The patients were required to sign an informed consent approved by the ethics committee of Tehran University of Medical Sciences, and to be available for monthly appointments up to 6 months. Exclusion criteria were histological sign of dysplasia, lichenoid drug reactions, and drug consumption in the past month, pregnancy, breast feeding or any kind of localized or systemic disease. Patients receiving immunosuppressive or immunomodulatory treatments or any kind of systemic or local drugs were either eliminated or asked to discontinue their treatment for a minimum of 1 month before entering the investigation. Patient who had lesions adjacent to amalgam filling were also excluded from the study sample.

Data associated with demographic, medical history, symptoms and clinical score of the lesion at the baseline were documented by an oral medicine specialist. Each patient received IMOD in the form of a daily single dose capsule $(400 \mathrm{mg}$ ) for 90 days. All participants were ex- amined at baseline, after 2 weeks and at months 1, 2, 3, 4, 5 and 6 in order to evaluate treatment efficacy. The clinical data or lesion score (LS) as an index of overall clinical improvement was scored as: 0 no lesion, 1 hyperkeratotic lesion, 2 atrophic areas $<1 \mathrm{~cm}, 3$ atrophic areas $>1 \mathrm{~cm}, 4$ erosive areas $<1 \mathrm{~cm}$, and 5 erosive areas $>1$ $\mathrm{cm}$. A zero value equates to being pain free, 1 indicates low degree of pain, 2 moderate pain, 3 severe pain, and 4 very severe pain [10].

A scaled tongue blade was used to assess the size of lesions [11]. Patients were given an oral examination, their lesions photographed, and then assessed against the numeric scale. Symptomatic response (SR) for each patient was calculated by subtracting the initial numeric scale from final score. Similarly, clinical response was estimated through subtraction LS values obtained in the first and last examination sessions. Positive and negative values were considered as improvement and deterioration, respectively.

The saliva level of TNF- $\alpha$ was measured at baseline, and at the end of treatment period. The unstimulated whole saliva (UWS) was collected between 9:00 a.m. and 12:00 a.m. The patients abstained from eating, drinking and smoking for at least 2 hours prior to the sampling. All subjects were requested to swallow first, tilt the head forward and then expectorate WUS into a sterile centrifugal tube without swallowing, for 5 - 15 minutes. The samples were then frozen until the time of analysis. TNF- $\alpha$ tests were performed by using ELISA kits (BioSource, Nevellis, Belgium).

\section{STATISTICAL ANALYSIS}

Analysis and comparison of symptomatic and clinical responses before and after treatment were performed using the Wilcoxon test. Paired student's t-test was used for comparison of TNF- $\alpha$ level before and after treatment. A P-value less than 0.05 was considered statistically significant. Statistical package for social science software (SPSS), used to analyze the data.

\section{RESULTS}

The study sample consisted of 21 women and 9 men with a mean age of $50.7 \pm 7.5$ years (range 35 - 66 years) and a total 112 lesions. The buccal mucosa was the most common site (47\%) for OLP, followed by gingiva (24\%), tongue $(22 \%)$, labial mucosa $(0.5 \%)$, and palate $(0.2 \%)$.

Approximately $85 \%$ of the lesions showed partial to complete clinical improvement at the end of the treatment period, and the results were also persistent in the three months follow-up period after drug therapy cessation (Table 1). 14\% of LSs had no change, and one person showed deterioration (Table 1). Analysis of Wilcoxon test showed that there was a significant difference 
in LS before and after treatment $(P<0.05)$ in the treatment and follow up periods (Table 1). OLP clinical scores decreased in almost all scoring groups. At the end of treatment, $69 \%$ of patients showed complete to partial SR (Table 2). A significant difference in SR was observed before and after treatment $(P<0.05)$. Numeric scale changes had significant improvement in all time point assessment through the trial course (Table 2).

Following treatment, the level of TNF- $\alpha$ (pg/ml) changed from $29.9 \pm 6.6$ to $39.7 \pm 8.3$ without statistically significant difference $(P=0.225)$ according to student's paired t-test (Figure 1).

\section{DISCUSSION}

The immunomodulatory mechanisms of IMOD are not clearly understood, but it can increase CD4+ lymphocyte count after 1 to 3 months treatment [12]. Its effect is also preserved for a long period after treatment cessation. It was primarily used in HIV infected patients, providing amelioration of AIDS manifestations. It has passed 3 phases of clinical trial without any considerable adverse effects. Considering the durability, safety and immunologic effects, it could be used in treatment of immunologic disorders [5]. Because of the immunologic na- ture of OLP [3], use of IMOD as an immunoregulator drug would appear reasonable for treatment outcome. This is the first clinical trial that evaluates the efficacy and compliance of this immunomodulator drug in OLP treatment.

In this study, erosive subtype changes from $45 \%$ of total lesions at base line to $7 \%$ after treatment. $33 \%$ of erosive lesions resolved completely, $20 \%$ converted to reticular, $42 \%$ to atrophic $<1 \mathrm{~cm}$ and $5 \%$ only had size reduction without subtype change. Converting erosive form to another type of lesion (atrophic and reticular form) with a decreased risk of malignant change can be considered an important outcome of this investigation. At the end of treatment, a significant improvement was seen in lesion clinical scores. $85 \%$ of lesions showed some degree of clinical improvement. In our recent study, we have evaluated the therapeutic efficacy of antioxidant- rich purslane (an herbal extract) on OLP lesions. The results showed that approximately $83 \%$ of lesions have been changed [11].

It was shown that in a randomized trial of pimecrolimus $1 \%$ cream (as case group) versus triamcinolone acetonide paste (control group) in OLP treatment, the mean clinical score at the base line was 2.4 , that decreased

Table 1. Clinical response.

\begin{tabular}{|c|c|c|c|c|c|c|c|}
\hline \multirow[b]{2}{*}{ Clinical response } & \multicolumn{4}{|c|}{ Treatment period } & \multicolumn{3}{|c|}{ Follow up period } \\
\hline & 2 weeks $\%$ & 1 month $\%$ & 2 months $\%$ & 3 months $\%$ & 4 months $\%$ & 5 months $\%$ & 6 months $\%$ \\
\hline+5 ( 5 degrees improvement $)$ & 0 & 0 & 2 & 5 & 7 & 8 & 10 \\
\hline +4 (4 degrees improvement) & 0 & 1 & 3 & 5 & 8 & 12 & 11 \\
\hline +3 (3 degrees improvement) & 4 & 4 & 8 & 11 & 13 & 14 & 14 \\
\hline+2 ( 2 degrees improvement $)$ & 30 & 41 & 41 & 41 & 35 & 34 & 34 \\
\hline +1 (1 degree improvement) & 19 & 18 & 26 & 23 & 23 & 20 & 17 \\
\hline 0 (no change) & 47 & 36 & 20 & 14 & 13 & 11 & 12 \\
\hline-4 (4 degrees worsening) & 0 & 0 & 0 & 0 & 0 & 1 & 1 \\
\hline-5 (5 degrees worsening) & 0 & 0 & 0 & 1 & 1 & 0 & 1 \\
\hline Median (range) & $+1(0,3)^{*}$ & $+1(0,4)^{*}$ & $+2(0,5)^{*}$ & $+2(-5,5)^{*}$ & $+2(-5,5)^{*}$ & $+2(-4,5)^{*}$ & $+2(-5,5)^{*}$ \\
\hline$P$ value & 0.001 & 0.001 & 0.001 & 0.001 & 0.001 & 0.001 & 0.001 \\
\hline
\end{tabular}

*Significant according to Wilcoxon test compared to before treatment $(P<0.05)$.

Table 2. Symptomatic response.

\begin{tabular}{|c|c|c|c|c|c|c|c|}
\hline \multirow[b]{2}{*}{ Symptomatic response } & \multicolumn{4}{|c|}{ Treatment period } & \multicolumn{3}{|c|}{ Follow up period } \\
\hline & 2 weeks $\%$ & 1 month \% & 2 months $\%$ & 3 months $\%$ & 4 months $\%$ & 5 months $\%$ & 6 months $\%$ \\
\hline+3 (3 degrees improvement) & 0 & 7 & 10 & 10 & 10 & 10 & 10 \\
\hline +2 (1 degrees improvement $)$ & 7 & 14 & 7 & 7 & 7 & 7 & 7 \\
\hline +1 (1 degree improvement) & 31 & 28 & 48 & 52 & 59 & 48 & 52 \\
\hline 0 (no change) & 59 & 48 & 35 & 31 & 24 & 35 & 31 \\
\hline-1 (1 degrees worsening) & 3 & 3 & 0 & 0 & 0 & 0 & 0 \\
\hline Median (range) & $0(-1,2)^{*}$ & $0(-1,3)^{*}$ & $+1(0,3)^{*}$ & $+1(0,3)^{*}$ & $+1(0,3)^{*}$ & $+1(0,3)^{*}$ & $+1(0,3)^{*}$ \\
\hline$P$ value & 0.005 & 0.002 & 0.001 & 0.001 & 0.001 & 0.001 & 0.001 \\
\hline
\end{tabular}

*Significant according to Wilcoxon test compared to before treatment $(P<0.05)$. 


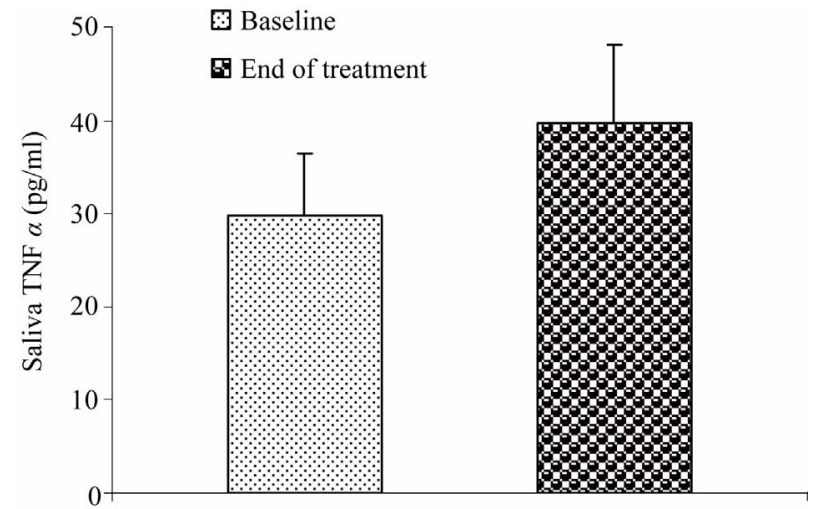

Figure 1. The unstimulated whole saliva concentration of TNF- $\alpha(\mathrm{pg} / \mathrm{ml})$ in baseline and end of the treatment with IMOD in patients with OLP; $P<0.05$ was considered statistically significant.

to 1.6 after two months of treatment [4]. Mean clinical score before treatment in this study was 3.38 , improving to 1.55 at the end of the three month treatment period, and 1.22 at the end of the follow-up period. It shows and emphasizes the highly durable therapeutic effect of IMOD after treatment cessation.

It was suggested methylene blue-mediated photodynamic therapy may be as a possible alternative treatment for OLP [13]. Thirteen persons with 26 lesions took part in their study. At the end of treatment, despite 4 reticular lesions, no complete remission was found in other scoring groups. In this investigation $33 \%$ of erosive lesions had complete remission and $20 \%$ converted to reticular form. $57 \%$ of reticular lesions disappeared after the treatment period. This shows the higher effectiveness of IMOD rather than photodynamic therapy in management of OLP lesion.

A downward shift of the numeric scale occurred in our patients at the end of the treatment period. The results were successfully preserved 3 months after treatment cessation in the follow up period. One study evaluated the efficacy of $0.1 \%$ dexamethasone oral rinse (3 times a day for 6 weeks) for treatment of erosive OLP. VAS (visual analog scale) value was decreased significantly at the end of the treatment period, but they did not assess the maintenance of treatment outcome in their study group [14]. However, it has been reported that one third of OLP patients treated with topical corticosteroids develop secondary candidiasis [15].

Until recent years, corticosteroids have been the mainstay of OLP treatment because of their anti-inflamma- tory and immunosuppressive effects. According to some reports TNF- $\alpha$ may be a good index for monitoring treat- ment response of corticosteroids, but not in any other drug subtypes. The mechanism of cytokine regulation by immunomodulator drugs has not been identified. But they potently inhibit TNF- $\alpha$ production by peripheral blood mononuclear cells, and enhance secretion of IL-2 and INF- $\gamma$ [16]. Despite significant improvement in patient symptoms and sign, no statistical differences were found in TNF- $\alpha$ level before and after treatment in this study. This finding may be explained by the assumption that TNF- $\alpha$ may be involved in the immunopathogenesis of OLP. Increasing differentiated $\mathrm{T}$ cell numbers after treatment by IMOD may be a possible explanation of the slight upward shift of TNF- $\alpha$ level.

Despite the devastating effect of TNF- $\alpha$, it has primary beneficial effects by initiation of inflammatory response, promoting angiogenesis and reparative process as well [10]. Corticosteroid drugs suppress both aspects of this double-edged sword cytokine. But an immunoregulator drug such as IMOD just regulates its action and promotes improvement in sign and symptom of OLP lesion, without deletion of the TNF- $\alpha$ beneficial effect that provides wound healing. It may be a possible explanation for the slight but statistically unimportant increase in TNF- $\alpha$ level after treatment with IMOD.

\section{CONCLUSIONS}

IMOD May be an efficacious choice of OLP treatment, but further controlled studies required to elucidate its real efficacy. TNF- $\alpha$ may not be a worthwhile tool in the follow-up in this study. The relationship of TNF- $\alpha$ level and other type 1 and type 2 cytokines in immunopathogenesis of OLP needs further study and may shed more light on its pathogenesis.

\section{REFERENCES}

[1] Ismail, S.B., Kumar, S.K. and Zain, R.B. (2007) Oral lichen planus and lichenoid reactions: Etiopathogenesis, diagnosis, management and malignant transformation. Journal of Oral Sciences, 49, 89-106. doi:10.2334/josnusd.49.89

[2] Agha-Hosseini, F., Mirzaii-Dizgah, I., Mikaili, S. and Abdollahi, M. (2009) Increased salivary lipid peroxidation in human subjects with oral lichen planus. International Journal of Dental Hygiene, 7, 246-250. doi:10.1111/j.1601-5037.2009.00365.x

[3] Greenberg S. (2008) Burket's oral medicine. 11th Edition, BC Decker Inc, Hamilton, 89-94.

[4] Gorouhi, F., Solhpour, A., Beitollahi, J.M., Afshar, S., Davari, P., Hashemi, P., Nassiri Kashani, M. and Firooz, A. (2007) Randomized trial of pimecrolimus cream versus triamcinolone acetonide paste in the treatment of oral lichen planus. Journal of the American Academy of Dermatology, 57, 806-813. doi:10.1016/i.jaad.2007.06.022

[5] Khairandish, P., Mohraz, M., Farzamfar, B., Abdollahi, M., Shahhosseiny, M.H. and Madani, H. (2009) Preclinical and phase 1 clinical safety of Setarud (IMOD ${ }^{\mathrm{TM}}$ ), a novel immunomodulator. 17, 148-156.

[6] Zhang, Y., Lin, M., Zhang, S., Wang, Z., Jiang, L., Shen, 
J., Bai, J., Gao, F., Zhou M. and Chen, Q. (2008) NF-kappaB-dependent cytokines in saliva and serum from patients with oral lichen planus: A study in an ethnic. Chinese population Cytokine, 41, 144-149. doi:10.1016/j.cyto.2007.11.004

[7] Pezelj-Ribaric S., Prso, I.B., Abram, M., Glazar, I., Brumini, G. and Simunovic-Soskic, M. (2004) Salivary levels of tumor necrosis factor-alpha in oral lichen planus. Mediators of Inflammation, 13, 131-133. doi:10.1080/09629350410001688530

[8] Banno, T., Gazel, A. and Blumenberg, M. (2004) Effects of tumor necrosis factor-alpha (TNF alpha) in epidermal keratinocytes revealed using global transcriptional profiling. The Journal of Biological Chemistry, 279, 3263332642. doi:10.1074/jbc.M400642200

[9] van der Meij, E. H. and van der Waal, I. (2003) Lack of clinicopathologic correlation in the diagnosis of oral lichen planus based on the presently available diagnostic criteria and suggestions for modifications. Journal of Oral Pathology \& Medicine, 32, 507-512. doi:10.1034/j.1600-0714.2003.00125.x

[10] Thongprasom, K., Luangjarmekorn, L., Sererat T. and Taweesap, W. (1992) Relative efficacy of fluocinolone acetonide compared with triamcinolone acetonide in treatment of oral lichen planus. Journal of Oral Pathology \& Medicine, 21, 456-458. doi:10.1111/j.1600-0714.1992.tb00974.x

[11] Agha-Hosseini, F., Borhan-Mojabi, K., Monsef-Esfahani, H.R., Mirzaii-Dizgah, I., Etemad-Moghadam S. and Karagah, A. (2010) Efficacy of Purslane in the treatment of oral lichen planus. Phytotherapy Research, 24, 240-244.
[12] Mohraz, M., Khairandish, P., Kazerooni, P.A., Davarpanah, M.A., Shahhosseiny, M.H., Mahdavian, B., Vaziry, S., Shahriary, S., Kamali, K., Khorram Khorshid, H.R., Heshmat, R., Farhadi M. and Gharibdoust, F. (2009) A clinical trial on the efficacy of IMOD in AIDS patients. 17, 277-284.

[13] Aghahosseini, F., Arbabi-Kalati, F., Fashtami, L.A., Djavid, G.E., Fateh M. and Beitollahi, J.M., (2006) Methylene blue-mediated photodynamic therapy: A possible alternative treatment for oral lichen planus. Lasers in Surgery and Medicine, 38, 33-38. doi:10.1002/lsm.20278

[14] Rhodus, N.L., Cheng, B., Bowles, W., Myers, S., Miller L. and Ondrey, F. (2006) Proinflammatory cytokine levels in saliva before and after treatment of (erosive) oral lichen planus with dexamethasone. Oral Diseases, 12, 112-116. doi:10.1111/j.1601-0825.2005.01165.x

[15] Lodi, G., Tarozzi, M., Sardella, A., Demarosi, F., Canegallo, L., Di Benedetto, D. and Carrassi, A. (2007) Miconazole as adjuvant therapy for oral lichen planus: a double-blind randomized controlled trial. British Journal of Dermatology, 156, 1336-1341. doi:10.1111/j.1365-2133.2007.07883.x

[16] Payvandi, F., Wu, L., Naziruddin, S.D., Haley, M., Parton, A., Schafer, P.H., Chen, R.S., Muller, G.W., Hughes, C.C. and Stirling, D.I. (2005) Immunomodulatory drugs (IMiDs) increase the production of IL-2 from stimulated $\mathrm{T}$ cells by increasing PKC-theta activation and enhancing the DNA-binding activity of AP-1 but not NF-kappaB, OCT-1, or NF-AT. Journal of Interferon \& Cytokine Research, 25, 604-616. doi:10.1089/jir.2005.25.604 